\title{
Human Biospecimens Come from People
}

\author{
Tom Tomlinson and Raymond G. De Vries
}

ABSTRACT Contrary to the revised Common Rule, and contrary to the views of many bioethicists and researchers, we argue that broad consent should be sought for anticipated later research uses of deidentified biospecimens and health information collected during medical care. Individuals differ in the kinds of risk they find concerning and in their willingness to permit use of their biospecimens for future research. For this reason, asking their permission for unspecified research uses is a fundamental expression of respect for them as persons and should be done absent some compelling moral consideration to the contrary. We examine three moral considerations and argue that each of them fails: that there is a duty of easy rescue binding on all, that seeking consent creates a selection bias that undermines the validity of biospecimen research, and that seeking and documenting consent will be prohibitively expensive.

KEY WORDS human research ethics, deidentified biospecimens, Common Rule, informed consent, broad consent, respect for persons

Tomlinson, T. and De Vries, R. G., Human Biospecimens Come from People, Ethics \& Human Research 41, no. 2 (2019): 22-28. D0I: 10.1002/ eahr.500009

W hen the Department of Health and Human Services (DHHS) issued its final rule that revised several provisions of the Common Rule governing research with humans, ${ }^{1}$ especially notable was one proposed rule not adopted: requiring at least broad consent to all future research uses of even deidentified biospecimens and health information collected from patients in the course of their medical care, or from individuals recruited to participate in medical research. Instead, the agency reaffirmed the existing rule allowing researchers to use these deidentified materials with no consent from the individual, since such uses did not count as research on "human subjects."

In an interesting coincidence, Oprah Winfrey released the film The Immortal Life of Henrietta Lacks shortly after the DHHS issued its final rule. The film is based on the book of the same name by Rebecca Skloot, ${ }^{2}$ which describes how researchers used-without Lacks's consent-cells that were obtained from procedures she underwent in the clinical setting to conduct research that led to important scientific breakthroughs in oncology.

The rejection of even a minimal consent requirement in the final revisions to the Common Rule was a decision supported by the majority of commentators who submitted comments to the DHHS about the proposed final revisions, ${ }^{3}$ and several bioethicists have endorsed the agency's decision. ${ }^{4}$ But we think they are wrong. Everyone should be asked for their broad consent when it is anticipated that their deidentified clinical specimens and data will be used in research.

The standard argument for not requiring consent is that once biospecimens and health information have been collected and strongly deidentified, it is highly unlikely that anything bad will happen to the "donor"5 no matter what is done with their biospecimens or associated data. Put this together with the potential of big data research for improving our understanding of disease and its prevention and treatment, add the assumption that any consent requirement would increase the cost or difficulty of such research, and the case against con- 
sent seems open and shut-an easy implication of the thoroughly risk-based ethical framework that lies at the heart of existing regulations in the United States. ${ }^{6}$

We believe these and other arguments against obtaining consent for research with biospecimens and associated data obtained in the clinical setting go wrong in a number of ways.

\section{PROTECTION OF VALUES AS IMPORTANT AS PROTECTION FROM MATERIAL HARMIS}

$\mathrm{T}$ he first problem is a misunderstanding of the nature of risk assessment: that the statistical risk of reidentification is all that matters to anyone worried about their sensitive information getting in the wrong hands. It's not. People can be reidentified using surprisingly little deidentified information. ${ }^{7}$ Donors need to know not only whether they can be reidentified; they also need to be able to decide whether the harms caused by reidentification are too high. The answer to that question depends on the type of research findings being protected and the implications for the person's welfare should those findings be disclosed, not just the statistical likelihood of reidentification.

If a biospecimen is used to discover whether tumors of the kind the patient had more readily metastasize, that's one thing. But if a biospecimen is used to find out if a genetic variation the patient has predicts a higher risk of some future illness, that's quite another, since this is exactly the sort of information that could be used to the patient's detriment. Some people might be much more worried than others about the risk of harm from disclosure of such information, and in fact such concerns can reduce willingness to contribute to genetic research under a broad consent approach. ${ }^{8}$

Yet without a consent requirement, those who most fear this possibility don't get the chance to say no to such research. The argument against requiring their consent for use of biospecimens obtained in the clinical setting is based on the assumption that whatever individual variation there may be in the weight attached to such concerns is morally irrelevant to whether consent should be sought. If "respect for persons" means, at a minimum, respect for and accommodation of individual variations in value judgments, then the argument fails to respect those persons whose materials and data are used without consent.
The argument that research on deidentified information requires no consent also turns on the idea that all that matters to people is the material harm that research might pose directly to them. But this is also untrue. When people deliberately donate their deidentified information to research, they care about a lot more than avoiding risk to themselves. Of course, those who donate want to do good by contributing to medical progress. But the corollary is that they don't want to do harm by contributing to something that they think is wrong or socially undesirable. This is why fair numbers of people, when asked, would not want their information made available to commercial drug companiesperhaps because of the understandable fear that the resulting drugs or treatments might be too costly and thus not available to all. ${ }^{9}$ Others would not want information

\section{Those who donate their deidentified} biospecimens want to do good by contributing to medical progress. But the corollary is that they don't want to do harm by contributing to something they think is wrong.

about them to be used in research identifying genetic predictors of a propensity for violence, out of concern that innocent people could be stigmatized. For some people, such concerns might be strong enough to outweigh their desire to contribute to medical research. ${ }^{10}$

It should not be surprising, then, that various studies confirm that a substantial minority of Americans are reluctant to give broad consent to unknown future research. ${ }^{11}$ A policy that does not give them the chance to say no shows no regard for their values and thus ignores a foundational principle that is supposed to govern the conduct of research: respect for persons.

The burden of proof is now on those who nevertheless want to ignore this principle, and that burden requires them to put forward countervailing considerations of sufficient weight. We address three such considerations: (1) that people have a duty to contribute to 
low-risk research and thus should not have the freedom to say no, (2) that asking for consent will create an unmanageable selection bias that would undermine the validity of big data research and would work to the disadvantage of racial and ethnic minorities, and (3) that asking for consent will be so administratively complex and expensive that it will divert funds from important research of benefit to public health.

\section{A DUTY OF EASY RESCUE}

Dorsdam-Mann and colleagues argue that "where - the risks involved in EHR [electronic health record] data sharing are or can be reduced to minimal, there is a duty of easy rescue to share EHR data for responsible and beneficial biomedical research." 12 What is the duty of easy rescue? The idea originates from a famous example of Peter Singer's: a man is walking by a shallow pond and sees a young child drowning in the middle. It would be a simple matter for the man to wade into the pond and save the child. The only risk would be getting his clothes muddy. It would be ethically monstrous to let the child die for such a trivial reason. ${ }^{13}$

From this example, Porsdam-Mann and colleagues derive a statement of the principle: "Duty of easy rescue. When the cost to $X$ of performing some action, $G$, is small, and the benefit to $\mathrm{Y}$ is large, then $\mathrm{X}$ ought to G." And then a collective version of it that can be applied to the big data research context: "Collective duty of easy recue: When the benefit of $\mathrm{G}$ to $\mathrm{Y}$ is large, and the cost of $\mathrm{G}$ to each of $\mathrm{X}^{1} \ldots \mathrm{X}^{\mathrm{n}}$ is small, then each of $\mathrm{X}^{1} \ldots \mathrm{X}^{\mathrm{n}}$ ought to G." ${ }^{\prime 4}$

In their example, every person could easily give just a few milliliters of blood, and if everyone did so, we could erase the blood shortage and save lives; hence there is a duty of each person to do so. In the same way, if we all ceded control of our biospecimens and data for health research, life-saving research could be done that would not be otherwise possible. Under this reasoning, there is, then, a duty to cede control that is strong enough to outweigh whatever autonomy rights we have over the uses of our biospecimens and data, and this justifies appropriating these for research without consent and contrary to the wishes of some.

Porsdam-Mann and colleagues acknowledge, of course, that the duty of easy rescue is not unconditional. As the rescue becomes more difficult or risky, the duty becomes weaker, to the point where it disappears completely, in acts of heroism. The duty does not justify any and all research uses of donors' biospecimens and data without consent. Research that posed some substantial risk of disclosure of sensitive information might not be justified by the duty, for example.

What, then, is the problem? It's that the weight of the duty of easy rescue depends not only on the level of risk to the rescuer but also on the level of harm that is averted for the rescued. The key question for this half of the duty is, what happens if I don't "rescue"? If the answer is "not much," then I don't have much of a duty. I don't have a duty to get my clothes muddy in the name of keeping the child's clothes from getting muddier. And if I waded in anyway, calling it a "rescue" would be a kind of joke.

The harm averted for the rescued is especially pertinent for gauging the collective duty of easy rescue, where my contribution to the outcome is miniscule. What harm is caused if I decide not to donate my few milliliters of blood? Nothing much, if anything at all. And the same is true if I decide not to donate my individual biospecimens and data to medical research. Unless I have a rare disease, my individual donation is the merest drop in the vast ocean of data being analyzed. No harm done. If there is no harm done by failing to "rescue," there is no duty to do so. And if there is no duty to do so, there is no countervailing moral duty of mine that overrides my right to be asked permission to use my biospecimens and data in research.

To avoid this trap, the argument might shift to a claim about fairness: it is not fair if I enjoy a benefit created by the sacrifices of others; in other words, I shouldn't be a free rider. A free rider is someone who enjoys a benefit made possible only by the collective action of others but avoids or refuses to do their own part. $^{15}$

One problem here is similar to the problem undermining the duty of easy rescue: surely the wrongfulness of my free riding is proportional to the sacrifices that others have made to create the good I now enjoy. If their sacrifices were great, then so is the wrongfulness of my free riding on them; but if their sacrifice was miniscule, then so is any wrong I've done them. Since the reasons for not requiring consent for later uses of deidentified biospecimens summarized earlier turn on the assump- 
tion that no sacrifice is asked of the donor, then I do those donors no wrong by enjoying the benefit of research they made possible.

However, what if everybody did that? Of course, the results could be terrible. But this doesn't show that each of us has an overriding duty to prevent this, because it will be prevented as long as enough people consentand as already indicated, most people will give broad consent to future uses of their biospecimens. If it were to turn out that most people won't give consent, insisting on their duty to do otherwise would do nothing to increase participation. Rather than stronger ethical exhortation, we would need better public education.

There is a final problem more specific to a free-riding argument. What if in fact I will not or cannot enjoy the collective good that has been created? In the present context, for example, what if I don't have good access to health care and so am less able to enjoy the fruits of research? Well, then I'm not free riding if I don't donate, because I'm not riding on the bus at all. If for reasons of poverty, poor education, lack of insurance, race, ethnicity, or so on, I have less access to medical advances, then the prohibition against free riding doesn't apply to me, leaving no justification for sidestepping my right to say no.

\section{SELECTION BIAS, RESEARCH VALIDITY, DISADVANTAGING SOME POPULATIONS}

This last point invites a reply that moves us to the I next argument against requiring consent: that seeking consent might itself create a selection bias that may disadvantage some populations. An assortment of studies has revealed that the willingness to provide a broad consent for future research on deidentified samples may vary by such things as race or ethnicity, income, educational levels, and sex. ${ }^{16}$ This poses the risk that the resulting data will contain a "selection bias" that under- or overrepresents certain populations. This may make it difficult to generalize research findings across the general population and to identify significant variations in subpopulations relevant to their disease risk, treatment response, and other important factors.

The argument, then, is that in order to avoid such biases, with their negative impact on the quality of research, and possibly on the distribution of the benefits of research across populations, we should not seek broad consent for unspecified uses of deidentified clinical biospecimens and data. ${ }^{17}$ If our goal is to fairly distribute the fruits of research across the population, we should not insist on a consent requirement that frustrates that goal.

For this argument to have enough weight to override the autonomy rights of donors, the frequency of selection bias needs to be widespread, its significance needs to be substantial rather than hypothetical, and there should be no other ways to address the problem. None of these are true.

Studies of differences in broad consent rates do not all point in the same directions. Some find significant differences by race; others do not. Some find significant differences by sex, or income, or educational level; others do not. The disparate findings led Kho et al. to conclude that although their systematic review of the literature suggested that "requirements for informed consent introduced a variety of biases into prospective observational studies using data from medical records, no systematic deviations occurred and the cause of the differences by age, sex, race, income, education, or health status that did emerge is unclear." ${ }^{18}$

Furthermore, we can determine readily enough whether a research sample is representative of the U.S. population. As Rothstein and Shoben point out, the fact that women (say) are underrepresented in a study does not demonstrate that this bias affects the relevance of the research results for women. For such selection bias to affect the validity of prospective studies, whether a woman gave (or withheld) her consent would have to be related to both the hypothesized cause and the hypothesized effect. The association between the consent decision and both cause and effect has to be very large to affect the validity of the relationship between the research variables, and so, in this case, it is unlikely to be a problem. What's more, there are standard and welldocumented techniques for detecting selection bias and adjusting for it. ${ }^{19}$

Even if selection bias were a significant problem adversely affecting the relevance of research results for disadvantaged minorities, it would be ironic to fix that injustice by perpetrating another, that is, not getting consent for research with biospecimens. This disenfranchises those who do not believe that the fruits of research will be available to them (often for good histori- 
cal and economic reasons) and who are most likely to not trust the scientific-medical enterprise or its experts.

The harder, more expensive, but more just solution is to directly address the causes for their skepticism. This effort could start by providing information about medical research on deidentified materials in language accessible to all. Cohn et al., for example, point out that in their survey of 51 biobanks in the United States, recruitment and enrollment materials were in English only, and most of the information provided exceeded the fifth-grade reading level. ${ }^{20}$ We can do better. One could imagine public information campaigns at the state, regional, or local levels explaining the nature and benefits of such research, as well as the importance of broad participation by all. Trust-and with it participation in research-could be further bolstered by community engagement efforts that recruit broadly representative advisory boards with meaningful roles advising on all aspects of recruitment and distribution of biospecimens and data. Of course, this is all going to cost more money. That brings us to the last objection.

\section{ADMINISTERING CONSENT WILL BE EXCESSIVELY DIFFICULT AND EXPENSIVE}

T he last major argument against requiring consent for research with biospecimens and data obtained in the clinical setting is that doing so would be administratively difficult and too expensive, wasting money better spent on research. As one commentator said about the DHHS's initial proposal for a consent requirement, "The proposed requirement for broad consent for future research associated with the NPRM's newly proposed definition would hinder research and substantially increase the cost and administrative workload while not enhancing human protections."21

Notice first that this argument simply dismisses out of hand the need to respect individual values of the sort we've described, since we are "wasting" money only if we think the cost protects something of little value. Surely, individual human autonomy is one of the things deserving "protection."

Note as well that the cost of a consent requirement depends very much on the source of the biopecimens and data and whether the consent is sought retroactively or proactively. For example, to get consent for research using annotated paraffin blocks of pathology samples with no available identifying information, a researcher would have to trace a sample back to the lab that prepared it, hoping it could then be linked to a person. If not impossible, this process would be prohibitively expensive. It would be theoretically possible to find and successfully communicate with a large population of previously identified patients or research participants for later permission to use their materials even if no contact had been maintained, but here too the costs would be prohibitive.

Prospective consent is quite another matter, and in fact, getting consent for the later research uses of clinical biospecimens is not likely to add much cost. Consent or not, robust research on deidentified clinical biospecimens requires a link between the individual and their information and biospecimens, created when these are deidentified and given a unique code. This link allows an individual to be alerted if some piece of research incidentally uncovers information important to that person's health or treatment. It also allows for the continuing collection and research use of updated medical information about the patient, increasing the value of big data by providing an ongoing medical "narrative" that supports prospective and not just retrospective research.

Creating and maintaining that link is where the real administrative effort and cost occur. Whether a particular patient has given consent becomes a check box in the electronic medical record. Whether a particular patient's information can then be made available for research is a question cheaply answered by a computer program.

The remaining question is how expensive it will be to administer the consent process and associated record keeping, and that will depend on how complex we make the regulatory requirements. Will we, for example, require individuals to "reconsent" every 10 years? ${ }^{22}$

Just what the regulatory requirements should be is not a question we are going to address here. We will just note that those who argue that since there is virtually no risk, one need not get consent at all are in no position to argue that if consent is sought, it needs to include all the elements of consent and the regulatory protections required when the research does pose risk. They should agree that since the risks associated with research on deidentified materials are very low, the consent form can 
be very simple, as illustrated by the one that Vanderbilt University Medical Center uses. ${ }^{23}$ Increased costs are inevitable, but may be manageable, and these costs are the price we should be willing to pay out of respect for those whose donations we would use.

\section{CONCLUSION}

$\mathrm{P}$ eople may have a variety of reasons for not agreeing to future research with their clinical biospecimens, based in their individual values and concerns. The fundamental Belmont principle of respect for persons requires that we offer patients a choice. Appeals to a duty to rescue, the risk of selection bias, or the potential additional costs of a consent option are not convincing or weighty enough to override that principle.

By asking for consent, researchers will show their respect both for the majority who are willing to say yes to research and the more cautious minority who have concerns about how their biospecimens may be used. In either case, gaining consent will make the public and biospecimen donors partners in scientific and medical progress-not unwitting conscripts. Yet with the growing reliance on biospecimen-based "big data" for medical research, the emergence of large corporate data brokers, ${ }^{24}$ and the revenue that universities, hospitals, and health systems can generate by providing these data, ${ }^{25}$ we may soon all be like Lacks-"participants" in research we know nothing about.

Tom Tomlinson, $\mathrm{PhD}$, is a professor at the Center for Ethics and Humanities in the Life Sciences at Michigan State University, and Raymond G. De Vries, $\mathbf{P h D}$, is a professor at the Center for Bioethics and Social Sciences in Medicine at the University of Michigan.

\section{ACKNOWLEDGMENTS}

Thanks to Caylin Rathburn-Smith, BA, for research assistance. This work was supported by grant 1 R01 HG007172$01 \mathrm{~A} 1$ from the National Human Genome Research Institute at the National Institutes of Health.

\section{REFERENCES}

1. "Federal Policy for the Protection of Human Subjects," Fed. Reg. (January 19, 2017), https://www.federalregister.gov/ documents/2017/01/19/2017-01058/federal-policy-for-theprotection-of-human-subjects\#h-3.

2. Skloot, R., The Immortal Life of Henrietta Lacks (New York: Broadway Books, 2011).
3. Council on Governmental Relations, "Analysis of Public Comments on the Common Rule NPRM," 2016, https://tinyurl.com/ydddtxkj.

4. Lynch, H. F., and M. N. Meyer, "Regulating Research with Biospecimens under the Revised Common Rule," Hastings Center Report 47, no. 3 (2017): 3-4; J. Menikoff, J. Kaneshiro, and I. Pritchard, "The Common Rule, Updated," New England Journal of Medicine 376, no. 7 (2017): 613-15; S. M. Rivera et al., "CTSA Institution Responses to Proposed Common Rule Changes: Did They Get What They Wanted?," Journal of Empirical Research on Human Research Ethics 12, no. 2 (2017): 79-86.

5. Given the position we argue for, it's difficult to settle on the right term for referring to those whose materials are being used. "Sources" is ambiguous between human and nonhuman sources and so obscures the person from whom materials were obtained. "Participants" is misleading, since after the data and specimens are generated, there is no more participation. "Donor" may subtly beg the question by suggesting that the person chose to provide materials. But since we think that is what should happen, "donor" is the term we will employ.

6. Tomlinson, T., "Respecting Donors to Biobank Research," Hastings Center Report 43, no. 1 (2013): 41-47.

7. Gymrek, M., et al., "Identifying Personal Genomes by Surname Inference," Science 339, no. 6117 (2013): 321-24.

8. Platt, J., et al., "Public Preferences regarding Informed Consent Models for Participation in Population-Based Genomic Research," Genetics in Medicine 16, no. 1 (2014): 11-18.

9. D’Abramo, F., J. Schildmann, and J. Vollmann, "Research Participants' Perceptions and Views on Consent for Biobank Research: A Review of Empirical Data and Ethical Analysis," BMC Medical Ethics 16 (2015): 60; Haddow, G., et al., "Tackling Community Concerns about Commercialization and Genetic Research: A Modest Interdisciplinary Proposal," Social Science and Medicine 64, no. 2 (2007): 272-82.

10. De Vries, R. G., et al., "The Moral Concerns of Biobank Donors: The Effect of Non-welfare Interests on Willingness to Donate," Life Sciences, Society and Policy 12 (2016): doi:10.1186/s40504-016-0036-4.

11. Simon, C. M., et al., "Active Choice but Not Too Active: Public Perspectives on Biobank Consent Models," Genetics in Medicine 13, no. 9 (2011): 821-31.

12. Porsdam-Mann, S., J. Savulescu, and B. J. Sahakian, "Facilitating the Ethical Use of Health Data for the Benefit of Society: Electronic Health Records, Consent and the Duty of Easy Rescue," Philosophical Transactions of the Royal Society A 374 (2016): doi:10.1098/rsta.2016.0130, p. 5 quoted.

13. Singer, P., "Famine, Affluence and Morality," Philosophy and Public Affairs 1 (1972): 229-43.

14. Porsdam-Mann, Savulescu, and Sahakian, "Facilitating the Ethical Use of Health Data for the Benefit of Society," 6.

15. Arneson, R. J., "The Principle of Fairness and Free-Rider 
Problems," Ethics 92, no. 4 (1982): 616-33.

16. Cohn, E. G., et al., "Self-Reported Race and Ethnicity of US Biobank Participants Compared to the US Census," Journal of Community Genetics 8, no. 3 (2017): 229-38; Hill, E. M., et al., "Let's Get the Best Quality Research We Can: Public Awareness and Acceptance of Consent to Use Existing Data in Health Research: A Review and Qualitative Study," BMC Medical Research Methodology 13 (2013): 72.

17. For example, see Lynch and Meyer, "Regulating Research with Biospecimens under the Revised Common Rule," 4.

18. Kho, M. E., et al., "Written Informed Consent and Selection Bias in Observational Studies Using Medical Records: Systematic Review, BMJ 338 (2009): doi: 10.1136/bmj.b866, p. 5 quoted.

19. Rothstein, M. A., and A. B. Shoben, "Does Consent Bias Research?," American Journal of Bioethics 13, no. 4 (2013): 27 37.

20. Cohn et al., "Self-Reported Race and Ethnicity of US Biobank Participants Compared to the US Census," 229.
21. Rivera et al., "CTSA Institution Responses to Proposed Common Rule Changes," 82.

22. Glimcher, L. H., "How Not to End Cancer in Our Lifetimes," Wall Street Journal, April 4, 2016; Secretary's Advisory Committee on Human Research Protections, "Extensive Resources Necessary to Obtain and Track Consent for Deidentified Biospecimens," section in "Attachment A: Recommendations NPRM," Office for Human Research Protections, content last reviewed March 9, 2016, https://www.hhs.gov/ ohrp/sachrp-committee/recommendations/2016-january5-recommendation-nprm-attachment-a.

23. "BioVu Consent Form," Vanderbilt University Medical Center, Institute for Clinical and Translational Research, January 14, 2019, https://victr.vanderbilt.edu/pub/ biovu/?sid=220.

24. BioIVT, https://www.bioivt.com/.

25. Ornstein, C., and K. Thomas, "Sloan Kettering's Cozy Deal with Start-Up Ignites a New Uproar," New York Times, September 20, 2018. 